M. Neubert: Theory of CP Violation in the B-Meson System (LP01)

\title{
THEORY OF CP VIOLATION IN THE B-MESON SYSTEM
}

\author{
MATTHIAS NEUBERT \\ Newman Laboratory of Nuclear Studies, Cornell University, Ithaca, NY 14853, USA \\ E-mail: neubert@mail.lns.cornell.edu
}

Recent developments in the theory of $\mathrm{CP}$ violation in the $B$-meson system are reviewed, with focus on the determination of $\sin 2 \beta$ from $B \rightarrow J / \psi K$ decays, its implications for tests of the Standard Model and searches for New Physics, and the determination of $\gamma$ and $\alpha$ from charmless hadronic $B$ decays.

\section{Introduction}

The phenomenon of $\mathrm{CP}$ violation is one of the most intriguing aspects of modern physics, with far-reaching implications for the microscopic world as well as for the macrocosmos. $\mathrm{CP}$ violation means that there is a fundamental difference between the interactions of matter and anti-matter, which in conjunction with the CPT theorem implies a microscopic violation of time-reversal invariance. $\mathrm{CP}$ violation is also responsible for the observed asymmetry in the abundance of matter and anti-matter in the Universe, which is a prerequisite for our existence.

The Standard Model (SM) of particle physics provides us with a parameterization of $\mathrm{CP}$ violation but does not explain its origin in a satisfactory way. In fact, $\mathrm{CP}$ violation may occur in three sectors of the SM: in the quark sector via the phase of the CabibboKobayashi-Maskawa (CKM) matrix, in the lepton sector via the phases of the neutrino mixing matrix, and in the strong interactions via the parameter $\theta_{\mathrm{QCD}}$. $\mathrm{CP}$ violation in the quark sector has been studied in some detail and is the subject of this talk. The nonobservation of $\mathrm{CP}$ violation in the strong interactions is a mystery (the "strong CP puzzle" whose explanation requires physics beyond the SM (such as a Peccei-Quinn symmetry, axions, etc.). $\mathrm{CP}$ violation in the neutrino sector has not yet been explored experimentally.

The discovery of $\mathrm{CP}$ violation in the $B$ system, as reported this summer by the
BaBarla and Belle ${ }^{3}$ Collaborations, is a triumph for the Standard Model. There is now compelling evidence that the phase of the CKM matrix correctly explains the pattern of CP-violating effects in mixing and weak decays of kaons, charm and beauty hadrons. Specifically, the CKM mechanism explains why $\mathrm{CP}$ violation is a small effect in $K-\bar{K}$ mixing $\left(\epsilon_{K}\right)$ and $K \rightarrow \pi \pi$ decays $\left(\epsilon^{\prime} / \epsilon\right)$, why $\mathrm{CP}$-violating effects in charm physics are below the sensitivity of present experiments, and why $\mathrm{CP}$ violation is small in $B-\bar{B}$ mixing $\left(\epsilon_{B}\right)$ but large in the interference of mixing and decay in $B \rightarrow J / \psi K\left(\sin 2 \beta_{\psi K}\right)$. The significance of the $\sin 2 \beta_{\psi K}$ measurement is that for the first time a large CP asymmetry has been observed, proving that $\mathrm{CP}$ is not an approximate symmetry of Nature. Rather, the smallness of $\mathrm{CP}$ violation outside the $B$ system simply reflects the hierarchy of CKM matrix elements.

Besides CP violation, the CKM mechanism explains a vast variety of flavorchanging processes, including leptonic decays, semileptonic decays (from which the magnitudes of most CKM elements are determined), nonleptonic decays, rare loop processes, and mixing amplitudes. The CKM matrix is a unitary matrix in flavor space, which relates the mass eigenstates of the down-type quarks with the interaction eigenstates that are involved in flavor-changing weak transitions. It has a hierarchical structure, and (with the standard phase conventions) the CP-violating phase appears in the smallest matrix elements, $V_{u b}$ and $V_{t d}$. The 


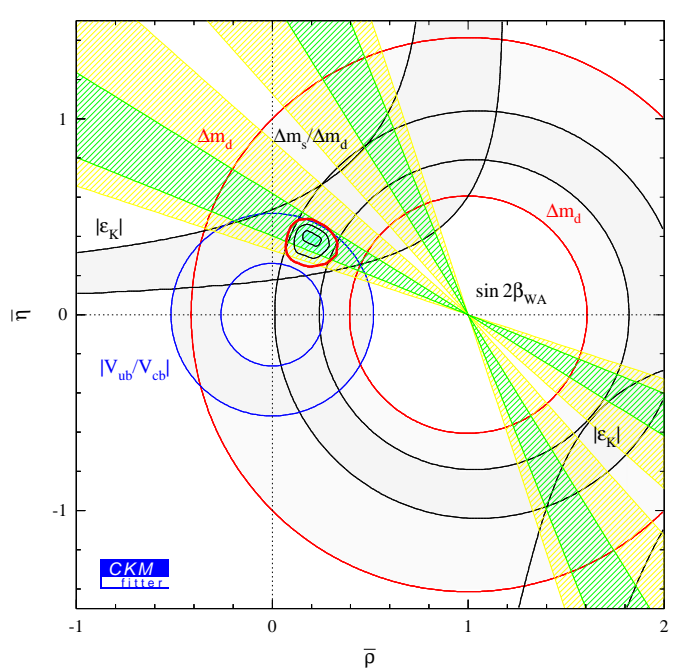

Figure 1. Summary of ständard constraints and global fit in the $(\bar{\rho}, \bar{\eta})$ plane.4

CKM matrix is described by four observable parameters, which can be taken to be the parameters of the Wolfenstein parameterization. Two of them, $\lambda=0.222 \pm 0.004$ and $A=0.83 \pm 0.07$ (at $95 \%$ confidence level) are rather accurately known, whereas the other two, $\bar{\rho}$ and $i \bar{\eta}$, are more uncertain. A convenient way of summarizing the existing information on $\bar{\rho}$ and $\bar{\eta}$ is to represent the unitarity relation $V_{u b}^{*} V_{u d}+V_{c b}^{*} V_{c d}+V_{t b}^{*} V_{t d}=0$ as a triangle in the complex plane. If the triangle is rescaled such that is has base of unit length, then the coordinates of the apex are given by $(\bar{\rho}, \bar{\eta})$. The angles of the unitarity triangle are related to $\mathrm{CP}$ violation. Figure 1 shows an example 1 of a recent global analysis of the unitarity triangle, combining measurements of $\left|V_{c b}\right|$ and $\left|V_{u b}\right|$ in semileptonic $B$ decays, $\left|V_{t d}\right|$ in $B-\bar{B}$ mixing, and the CPviolating phase of $V_{t d}^{2}$ in $K-\bar{K}$ mixing and $B \rightarrow J / \psi K$ decays. The values obtained at $95 \%$ confidence level are $\bar{\rho}=0.21 \pm 0.12$ and $\bar{\eta}=0.38 \pm 0.11$. The corresponding results for the angles of the unitarity triangle are $\sin 2 \beta=0.74 \pm 0.14, \sin 2 \alpha=-0.14 \pm 0.57$, and $\gamma=(62 \pm 15)^{\circ}$. These studies have established the existence of a $\mathrm{CP}$-violating phase
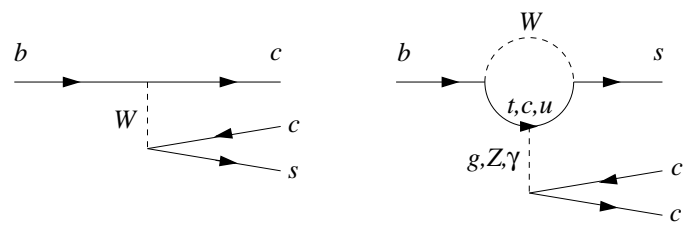

Figure 2. Tree and penguin topologies in $B \rightarrow J / \psi K$ decays.

in the top sector of the CKM matrix, i.e., the fact that $\operatorname{Im}\left(V_{t d}^{2}\right) \propto \bar{\eta} \neq 0$.

\section{Determination of $\sin 2 \beta_{\psi K}$}

In decays of neutral $B$ mesons into a $\mathrm{CP}$ eigenstate $f_{\mathrm{CP}}$, an observable $\mathrm{CP}$ asymmetry can arise from the interference of the amplitudes for decays with an without $B-\bar{B}$ mixing, i.e., from the fact that the amplitudes for $B^{0} \rightarrow f_{\mathrm{CP}}$ and $B^{0} \rightarrow \bar{B}^{0} \rightarrow f_{\mathrm{CP}}$ must be added coherently. The resulting timedependent asymmetry is given by

$$
\begin{aligned}
& A_{\mathrm{CP}}(t)=\frac{\Gamma\left(\bar{B}^{0}(t) \rightarrow f_{\mathrm{CP}}\right)-\Gamma\left(B^{0}(t) \rightarrow f_{\mathrm{CP}}\right)}{\Gamma\left(\bar{B}^{0}(t) \rightarrow f_{\mathrm{CP}}\right)+\Gamma\left(B^{0}(t) \rightarrow f_{\mathrm{CP}}\right)} \\
& =\frac{2 \operatorname{Im}(\hat{\lambda})}{1+|\hat{\lambda}|^{2}} \sin \left(\Delta m_{d} t\right)-\frac{1-|\hat{\lambda}|^{2}}{1+|\hat{\lambda}|^{2}} \cos \left(\Delta m_{d} t\right)
\end{aligned}
$$

where $\hat{\lambda}=e^{i \phi_{d}} \bar{A} / A, \phi_{d}$ is the $B-\bar{B}$ mixing phase (which in the SM equals $-2 \beta$ ), and $A(\bar{A})$ denotes the $B^{0}\left(\bar{B}^{0}\right) \rightarrow f_{\mathrm{CP}}$ decay amplitude. If the amplitude is dominated by a single weak phase $\phi_{A}$, then $|\hat{\lambda}| \simeq 1$ and

$$
A_{\mathrm{CP}}(t) \simeq \eta_{f_{\mathrm{CP}}} \sin \left(\phi_{d}-2 \phi_{A}\right) \sin \left(\Delta m_{d} t\right),
$$

where $\eta_{f_{\mathrm{CP}}}= \pm 1$ is the $\mathrm{CP}$ signature of the final state.

The "golden mode" $B \rightarrow J / \psi K_{S}$ (for which $\left.\eta_{\psi K_{S}}=-1\right)$ is based on $b \rightarrow c \bar{c} s$ transitions, which in the SM can proceed via tree or penguin topologies, as shown in Figure 2. To an excellent approximation the decay amplitude for this process is real. A weak phase is introduced only through components of the up- and top-quark penguin diagrams that are strongly CKM suppressed. Parametrically, the "penguin pollution" to 
the weak phase from these effects is of order $\Delta \phi_{A} \sim \lambda^{2}(P / T) \sim 1 \%$, where $P / T \sim 0.2$ is the tree-to-penguin ratio. It follows that $A_{\mathrm{CP}}(t) \simeq \sin 2 \beta \sin \left(\Delta m_{d} t\right)$ with an accuracy of about $1 \%$.

This summer, the BaBar and Belle Collaborations have presented measurements of $\sin 2 \beta_{\psi K}$ with unprecedented precision The results are $0.59 \pm 0.14 \neq 0.05(\mathrm{BaBar})$ and $0.99 \pm 0.14 \pm 0.06$ (Belle 3 ), which when combined with earlier determinations lead to the new world average $\sin 2 \beta_{\psi K}=0.79 \pm$ 0.10. The expectation obtained from the global analysis of the unitarity triangle (leaving aside earlier $\sin 2 \beta_{\psi_{K}}$ measurements) was $\sin 2 \beta=0.68 \pm 0.21$ at $95 \%$ confidence level, 1 in good agreement with the new data.

The above discussion relies on the SM and could be upset if there existed a New Physics contribution to $B-\bar{B}$ mixing, or a new contribution to the $b \rightarrow c \bar{c} s$ transition amplitude with a nonzero weak phase $\phi_{\mathrm{NP}}$. The latter case is, however, rather unlikely, since such a nonstandard contribution could hardly compete with the large tree-level amplitude of the SM. Otherwise there should be signals of direct $\mathrm{CP}$ violation in decays such as $B^{ \pm} \rightarrow J / \psi K^{ \pm}$, and there should be other $b \rightarrow q \bar{q} s$ New Physics contributions of similar strength $\left(\sim \lambda^{2}\right)$, which would upset the phenomenology of charmless hadronic decays such as $B \rightarrow \pi K, \pi \pi$, etc. Hence, it appears safe to assume that even in the presence of New Physics the time-dependent $\mathrm{CP}$ asymmetry observed in $B \rightarrow J / \psi K$ decays measures the $B-\bar{B}$ mixing phase, so that $\sin 2 \beta_{\psi K}=-\sin \phi_{d}$. The good agreement of the measured phase with the SM prediction suggests that at least the dominant part of the $B-\bar{B}$ mixing phase is due to the phase of the CKM matrix element $V_{t d}$. (This ignores the possibility of an accidental agreement made possible by a discrete ambiguity. In other words, there could still be a large New Physics contribution to $B-\bar{B}$ mixing such that $\phi_{d} \approx \pi+2 \beta$.)

\section{The Quest for New Physics}

While we are amazed by the workings of the SM, some theorists will be disappointed by the fact that $\sin 2 \beta_{\psi K}$ does not show a hint for New Physics. In many extensions of the SM it would have been quite possible (sometimes even required) for the $B-\bar{B}$ mixing phase to differ from its SM value 6.60 For instance, potentially large effects could arise in models with iso-singlet down-type quarks and tree-level flavor-changing neutral currents, 11 left-right symmetric models with spontaneous CP violation 12.13, 14 (which are now excluded by the data), and SUSY models with extended minimal flavor violation.15 On the contrary, only small modifications of $\sin 2 \beta_{\psi K}$ are allowed in a class of models with so-called minimal flavor violation $14,6,17$ for which one can derive the bounds $0.52<$ $\sin 2 \beta_{\psi K}<0.78$.

Much like the stunning success of the SM in explaining electroweak precision data, the observed lack of deviations from the predictions of the CKM model is, to some extent, surprising. Recall that the CKM mechanism neither explains the baryon asymmetry in the Universe nor offers a clue as to why $\mathrm{CP}$ violation does not occur in the strong interactions. There are good arguments suggesting that the stability of the electroweak scale will be explained by some New Physics at or below the $\mathrm{TeV}$ scale. But virtually all extensions of the SM contain many new CPviolating couplings. For instance, a minimal, unconstrained (i.e., not fine-tuned) SUSY extension introduces 43 complex couplings in addition to the CKM phase. The fact that experiments have not shown any trace of nonstandard $\mathrm{CP}$ violation is puzzling and creates what one may call the "CP problem". It is not unlikely that the "decoupling" of nonstandard CP violation effects is linked to the decoupling of New Physics in the sector of electroweak symmetry breaking. In that sense, the $B$ factories offer a complementary 
strategy for probing $\mathrm{TeV}$-scale physics. Like with the search for the explanation of electroweak symmetry breaking, the fact that we have not yet found New Physics in the flavor sector does not mean that it is not there, it just means we have to look harder. Hence, the strategy should be to keep searching for (probably small) deviations from the CKM paradigm with ever more precise measurements.

Given what we have learned about flavor physics in the kaon and beauty systems, there is still plenty of room for New Physics effects in both mixing and weak decays, and there is reason to hope that departures from the predictions of the SM may be discovered soon. Following is a list of options for discovering some potentially large New Physics effects:

1. Check if the strength of $B_{s}-\bar{B}_{s}$ mixing is correctly predicted by the $\mathrm{SM}$, i.e., confirm or disprove that $\Delta m_{s} \approx(17 \pm 3) \mathrm{ps}^{-1}$.

2. Measure the CP-violating phase $\gamma=$ $\arg \left(V_{u b}^{*}\right)$ in the bottom sector and check if it agrees with the value inferred from the standard global analysis of the unitarity triangle using measurements of $\mathrm{CP}$ violation in the top sector. The current prejudice that $\gamma$ must be less than $90^{\circ}$ relies on the assumption that $B_{s}-\bar{B}_{s}$ mixing is not affected by New Physics. A first opportunity for probing $\gamma$ directly is offered by the analysis of charmless hadronic $B$ decays, as discussed below.

3. Probe for New Physics in a variety of rare decay processes (proceeding through penguin and box diagrams in the SM). Some examples are:

- Check if $\sin 2 \beta_{\phi K}=\sin 2 \beta_{\psi K}$. If not, this would be clear evidence for New Physics in $b \rightarrow s \bar{s} s$ penguin transitions. 18

- Look for a direct CP asymmetry in $B \rightarrow$ $X_{s} \gamma$ decays. Any signal exceeding the level of $1 \%$ would be a clear sign of New Physics in radiative penguin decays. 19

- Check if $\gamma$ measured in the penguin-
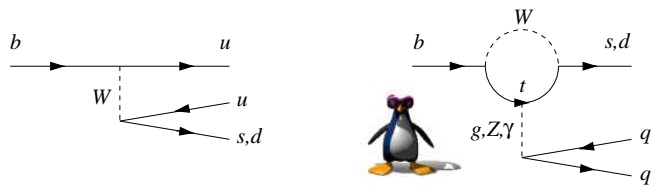

Figure 3. Tree and penguin topologies in charmless hadronic $B$ decays.

dominated decays $B \rightarrow \pi K$ agrees with $\gamma$ extracted from pure tree-processes such as $B \rightarrow D K$ and $B \rightarrow D^{*} \pi .20 .21$

- Look for New Physics in $B \rightarrow K l^{+} l^{-}$decays, for instance by testing the prediction of a form-factor zero in the forwardbackward asymmetry.222324

4. Measure the branching ratios for the very rare kaon decays $K^{ \pm} \rightarrow \pi^{ \pm} \nu \bar{\nu}$ and $K_{L} \rightarrow$ $\pi^{0} \nu \bar{\nu}$, which allow for an independent construction of the unitarity triangle.25

5. Search for New Physics in $D-\bar{D}$ mixing and charm weak decays.

6. Continue to look for $\mathrm{CP}$ violation outside of flavor physics by probing electric dipole moments of the neutron and electron.

\section{Charmless Hadronic Decays}

After establishing the existence of a weak phase in the top sector by showing that $\operatorname{Im}\left(V_{t d}^{2}\right) \neq 0$, the next step in testing the CKM paradigm must be to explore the CPviolating phase in the bottom sector, $\gamma=$ $\arg \left(V_{u b}^{*}\right)$. In the SM the two phases are, of course, related to each other. However, as discussed above there is still much room for New Physics to affect the magnitude of flavor violations in both mixing and weak decays.

Common lore says that measurements of $\gamma$ are difficult. Several theoretically clean determinations of this phase from pure tree processes such as $B \rightarrow D K^{26}$ and $B \rightarrow D^{*} \pi{ }^{27}$ have been suggested, which are extremely challenging experimentally. Likewise, measurements of $\beta+\gamma=\pi-\alpha$ using isospin 


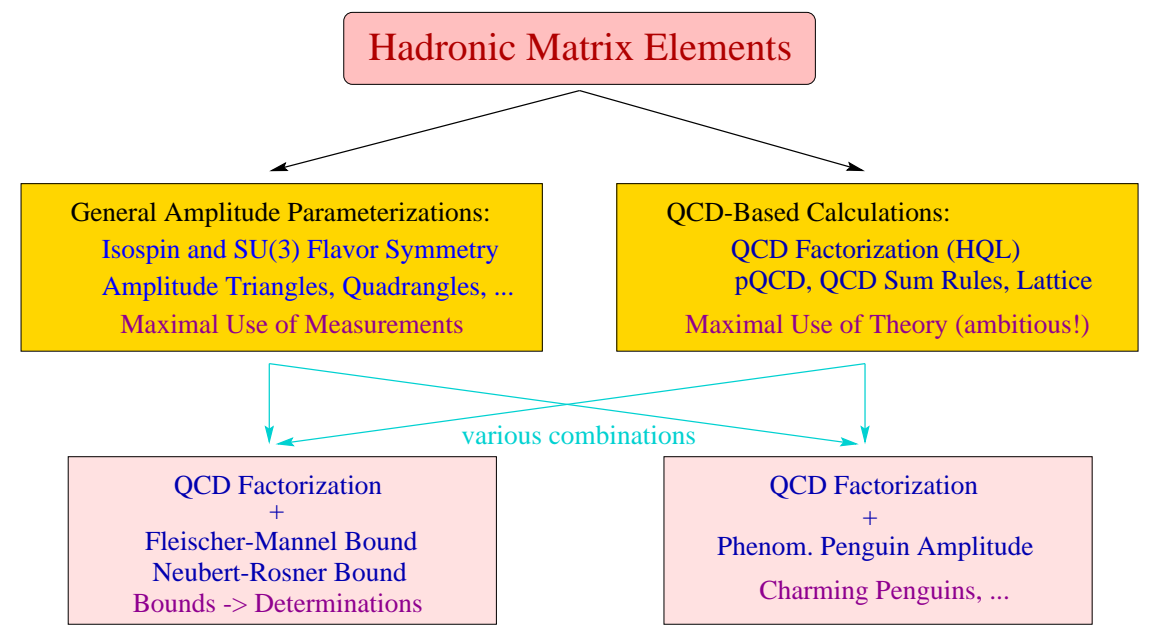

Figure 4. Strategies used to determine hadronic matrix elements entering charmless hadronic $B$ decays.

analysis in $B \rightarrow \pi \pi$ decays 4 or Dalitz plot analysis in $B \rightarrow \pi \pi \pi$ decays 29 are very difficult. It is more accessible experimentally to probe $\gamma$ via the sizeable tree-penguin interference in charmless hadronic decays such as $B \rightarrow \pi K$ and $B \rightarrow \pi \pi$. The basic decay topologies contributing to these modes are shown in Figure 3. Experiment shows that the tree-to-penguin ratios in the two cases are roughly $|T / P|_{\pi K} \approx 0.2$ and $|P / T|_{\pi \pi} \approx 0.3$, indicating a sizeable amplitude interference. It is important that the relative weak phase between the two amplitudes can be probed not only via $\mathrm{CP}$ asymmetry measurements $(\sim \sin \gamma)$, but also via measurements of CPaveraged branching fractions $(\sim \cos \gamma)$.

Extracting information about CKM parameters from the analysis of nonleptonic $B$ decays is a challenge to theory, since it requires some level of control over hadronic physics, including strong-interaction phases. Figure 1 illustrates the two main strategies for tackling the problem of hadronic matrix elements: general amplitude parameterizations avoiding any dynamical input on one hand, and QCD-based calculations on the other. In the first approach, decay amplitudes are cataloged according to the flavor topologies that can contribute to a given decay process, such as tree topologies, penguin topologies, annihilation topologies, etc 30 The various topologies can be related to renormalization-group invariant combinations of operator matrix elements of the effective weak Hamiltonian, 31 but no attempt is made to calculate these matrix elements from first principles. Instead, isospin symmetry or, more generally, SU(3) flavor symmetry is used to obtain relations between the various amplitudes in different decay modes. Experimental data is then used to determine as many hadronic parameters as possible. This leads to the well-known amplitude triangle (and quadrangle) constructions, from which $\mathrm{CP}$-violating phases can be extracted (modulo discrete ambiguities) in the limit of exact flavor symmetry 32 QCD-based calculations of hadronic matrix elements are more ambitious in that they aim at an understanding of the underlying strong-interaction dynamics from first principles. Factorization theorems (such as the QCD factorization apprgach 33 34 and the hard-scattering approach 35 , 36 ) attack this problem by exploiting the heavy-quark limit. Other schemes, such as QCD sum rules and lattice QCD, are applicable to a wider class of processes, including hadronic decays of light mesons. Unfortunately, at present 
these approaches still face tremendous technical difficulties when attempting the calculation of nonleptonic decay amplitudes. A very promising strategy is to combine the results obtained using amplitude parameterization with some dynamical information derived from QCD-based calculations. For instance in that way model-independent bounds 37.38 on the CP-violating phase $\gamma$ can be turned into determinations of $\gamma$ that are subject to only very small theoretical uncertainties.

\section{QCD Factorization}

The statement that hadronic weak decay amplitudes simplify greatly in the heavy-quark limit $m_{b} \gg \Lambda_{\mathrm{QCD}}$ will not surprise those who have followed the dramatic advances in the theoretical understanding of $B$ physics during the past decade. Many areas of $B$ physics, from spectroscopy to exclusive semileptonic decays to inclusive rates and lifetimes, can now be systematically analyzed using heavyquark expansions. Yet, the more complicated exclusive nonleptonic decays have long resisted theoretical progress. The technical reason is that, whereas in most other applications of heavy-quark expansions one proceeds by integrating out heavy fields (leading to local operator product expansions), in the case of nonleptonic decays the large scale $m_{b}$ enters as the energy carried by light fields. Therefore, in addition to hard and soft subprocesses collinear degrees of freedom become important. This complicates the understanding of hadronic decay amplitudes using the language of effective field theory. (Yet, significant progress towards an effective fieldtheory description of nonleptonic decays has been made recently with the establishment of a "collinear-soft effective theory" 39 The reader is referred to these papers for more details on this development.)

The importance of the heavy-quark limit is linked to the idea of color transparency. 40.41 .42 A fast-moving light meson (such as a pion) produced in a point-like source (a local operator in the effective weak Hamiltonian) decouples from soft QCD interactions. More precisely, the couplings of soft gluons to such a system can be analyzed using a multipole expansion, and the leading contribution (from the color dipole) is suppressed by a power of $\Lambda_{\mathrm{QCD}} / m_{b}$. The QCD factorization approach provides a systematic implementation of this idea.33,34 It yields rigorous results in the heavy-quark limit, which are valid to leading power in $\Lambda_{\mathrm{QCD}} / m_{b}$ but to all orders of perturbation theory. Having obtained control over nonleptonic decays in the heavy-quark limit is a tremendous advance. We are now able to talk about power corrections to a well-defined and calculable limiting case, which captures a substantial part of the physics in these complicated processes.

The workings of QCD factorization can be illustrated with the cartoons shown in Figure 5. The first graph shows the wellknown concept of an effective weak Hamiltonian obtained by integrating out the heavy fields of the top quark and weak gauge bosons from the SM Lagrangian. This introduces new effective interactions mediated by local operators $O_{i}(\mu)$ (typically four-quark operators) multiplied by calculable running coupling constants $C_{i}(\mu)$ called Wilson coefficients. This reduction in complexity (nonlocal heavy particle exchanges $\rightarrow$ local effective interactions) is exact up to corrections suppressed by inverse powers of the heavy mass scales. The resulting picture at scales at or above $m_{b}$ is, however, still rather complicated, since gluon exchange is possible between any of the quarks in the external meson states. Additional simplifications occur when the renormalization scale $\mu$ is lowered below the scale $m_{b}$. Then color transparency comes to play and implies systematic cancellations of soft and collinear gluon exchanges. As a result, all "nonfactorizable" exchanges, i.e., gluons connecting the light meson at the "upper" vertex to the remaining mesons, are 

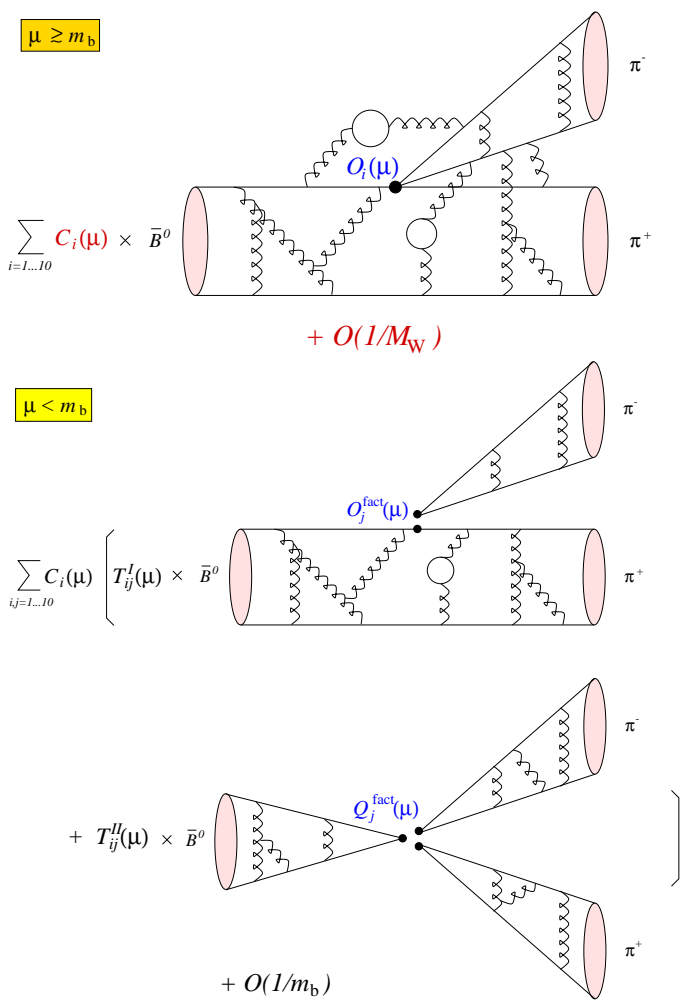

Figure 5. Factorization of short- and long-distance contributions in hadronic $B$ decays. Upper: Factorization of short-distance effects into Wilson coefficients of the effective weak Hamiltonian. Lower: Factorization of hard "nonfactorizable" gluon exchanges into hard-scattering kernels (QCD factorization).

dominated by virtualities of order $m_{b}$ and can be calculated. Their effects are absorbed into a new set of running couplings $T_{i j}^{I, I I}(\mu)$ called hard-scattering kernels, as shown in the two lower graphs. What remains are "factorized" four-quark and six-quark operators $O_{j}^{\mathrm{fact}}(\mu)$ and $Q_{j}^{\mathrm{fact}}(\mu)$, whose matrix elements can be expressed in terms of form factors, decay constants and light-cone distribution amplitudes. As before, the reduction in complexity (local four-quark operators $\rightarrow$ "factorized" operators) is exact up to corrections suppressed by inverse powers of the heavy scale, now set by the $b$-quark mass.

The factorization formula is valid in all cases where the meson at the "upper" vertex is light, meaning that its mass is much smaller than the $b$-quark mass. The second term in the factorization formula (the term involving "factorized" six-quark operators) gives a power-suppressed contribution when the final-state meson at the "lower" vertex is a heavy meson (i.e., a charm meson), but its contribution is of leading power if this meson is also light.

Factorization is a property of decay amplitudes in the heavy-quark limit. Given the magnitudes of "nonfactorizable" effects seen in kaon, charm and beauty decays, there can be little doubt about the relevance of the heavy-quark limit to understanding nonleptonic processes 43 Yet, for phenomenological applications it is important to explore the structure of at least the leading power-suppressed corrections. While no complete classification of such corrections has been achieved to date, several classes of power-suppressed terms have been analyzed and their effects estimated. They include "chirallyenhanced" power corrections, 33 weak annihilation contributions, 34,44 and power corrections due to nonfactorizable soft gluon exchange.46.47. 0 With the exception of the "chirally-enhanced" terms, no unusually large power corrections (i.e., corrections exceeding the naive expectation of $5-10 \%$ ) have been identified so far. Nevertheless, it is important to refine and extend the estimates of power corrections. Fortunately, the QCD factorization approach makes many testable predictions. Ultimately, therefore, the data will give us conclusive evidence on the relevance of power-suppressed effects. Many tests can already be performed using existing data.

\subsection{Tests of Factorization in $B \rightarrow D^{(*)} L$}

In $B$ decays into a heavy-light final state, when the light meson is produced at the "upper" vertex, the factorization formula assumes its simplest form. Then only the form factor term (the first graph in the lower 
portion of Figure 5) contributes at leading power. This is also the place where QCD factorization is best established theoretically. The systematic cancellation of soft and collinear singularities has been demonstrated to all orders in perturbation theory 34,49 and in the "large- $\beta_{0}$ limit" of QCD it has been shown that the hard-scattering kernels are free of power-like endpoint singularities as one of the quarks in the light meson becomes a soft parton. whether such a smooth behavior persists in higher orders of full QCD.)

In the case of the decays $\bar{B}^{0} \rightarrow D^{(*)+} L^{-}$, where $L$ denotes a light meson, the flavor content of the final state is such that the light meson can only be produced at the "upper" vertex, so factorization applies. One finds that process-dependent "nonfactorizable" corrections from hard gluon exchange, though present, are numerically very small. All nontrivial QCD effects in the decay amplitudes are then described by a quasiuniversal coefficient $\left|a_{1}\left(D^{(*)} L\right)\right|=1.05 \pm$ $0.02+O\left(\Lambda_{\mathrm{QCD}} / m_{b}\right) .34$ For a given decay channel this coefficient can be determined experimentally from the ratio 40

$$
\begin{gathered}
\frac{\Gamma\left(\bar{B}^{0} \rightarrow D^{*+} L^{-}\right)}{d \Gamma\left(\bar{B}^{0} \rightarrow D^{*+} l^{-} \nu\right) /\left.d q^{2}\right|_{q^{2}=m_{L}^{2}}} \\
=6 \pi^{2}\left|V_{u d}\right|^{2} f_{L}^{2}\left|a_{1}\left(D^{(*)} L\right)\right|^{2} .
\end{gathered}
$$

Using CLEO data one obtains $\left|a_{1}\left(D^{*} \pi\right)\right|=$ $1.08 \pm 0.07,\left|a_{1}\left(D^{*} \rho\right)\right|=1.09 \pm 0.10$, and $\left|a_{1}\left(D^{*} a_{1}\right)\right|=1.08 \pm 0.11$, in good agreement with the theoretical prediction. This is a first indication that power corrections in these modes are under control, but more precise data are required for a firm conclusion. For other tests of factorization in $B$ decays to heavy-light final states the reader is referred to recent literature.34.59,51

The experimental observation of unexpectedly large rates for color-suppressed decays 2 . 3 such as $\bar{B}^{0} \rightarrow D^{0(*)} \pi^{0}$ has attracted some attention. QCD factorization does not allow us to calculate the amplitudes for these processes in a reliable way. It predicts that these amplitudes are powersuppressed with respect to the corresponding $\bar{B}^{0} \rightarrow D^{+(*)} \pi^{-}$amplitudes, but only by one power of $\Lambda_{\mathrm{QCD}} / m_{c}$. Specifically, the prediction is that a certain ratio of isospin amplitudes approaches unity in the heavy-quark limit: $A_{1 / 2} /\left(\sqrt{2} A_{3 / 2}\right)=1+O\left(\Lambda_{\mathrm{QCD}} / m_{c}\right)$. This scaling law is respected by the experimental data, which give $A_{1 / 2} /\left(\sqrt{2} A_{3 / 2}\right)=$ $(0.70 \pm 0.11) e^{ \pm i(27 \pm 7)^{\circ}}$ for $B \rightarrow D \pi$ and $(0.72 \pm 0.08) e^{ \pm i(21 \pm 8)^{\circ}}$ for $B \rightarrow D^{*} \pi$. $43 \mathrm{~A}$ rough theoretical estimate of the amplitude ratio, $A_{1 / 2} /\left(\sqrt{2} A_{3 / 2}\right) \approx 0.75 e^{-15^{\circ} i}$, had been obtained prior to the observation of the color-suppressed decays. 34 It anticipated the correct order of magnitude of the deviation from the heavy-quark limit.

\subsection{Tests of Factorization in $B \rightarrow K^{*} \gamma$}

The QCD factorization approach not only applies to nonleptonic decays, but also to other exclusive processes such as $B \rightarrow V \gamma$ and $B \rightarrow V l^{+} l^{-}$, where $V=K^{*}, \rho, \ldots$ is a vector meson.24 54 The resulting factorization formula is similar (but simpler) to that for $B$ decays into two light mesons. The study of exclusive radiative transitions therefore not only extends the range of applicability of the method, it also provides a new testing ground for the factorization idea.

Interestingly, the analysis of isospinbreaking effects in radiative $B$ decays, as measured by the quantity 55.56 .57

$$
\begin{aligned}
\Delta_{0-} & \equiv \frac{\Gamma\left(\bar{B}^{0} \rightarrow \bar{K}^{* 0} \gamma\right)-\Gamma\left(B^{-} \rightarrow \bar{K}^{*-} \gamma\right)}{\Gamma\left(\bar{B}^{0} \rightarrow \bar{K}^{* 0} \gamma\right)+\Gamma\left(B^{-} \rightarrow \bar{K}^{*-} \gamma\right)} \\
& =0.11 \pm 0.07
\end{aligned}
$$

gives a direct probe of power corrections to the factorization formula, since such effects are absent in the heavy-quark limit. A theoretical analysis of the leading power-suppressed contributions gives $\Delta_{0-}=\left(8.0_{-3.2}^{+2.1}\right) \% \times\left(0.3 / T_{1}^{B \rightarrow K^{*}}\right) 58$ where $T_{1}^{B \rightarrow K^{*}} \sim 0.3$ is a $B \rightarrow K^{*}$ form factor. The 
largest contribution comes from an annihilation contribution involving the penguin operator $O_{6}$ in the effective weak Hamiltonian. As a result, the quantity $\Delta_{0-}$ is a sensitive probe of the magnitude and sign of the ratio $C_{6} / C_{7 \gamma}$ of Wilson coefficients.

The theoretical prediction for $\Delta_{0-}$ is in agreement with the current experimental value. If this agreement persists as the data become more precise, this would not only test the penguin sector of the effective weak Hamiltonian but also provide a quantitative test of factorization at the level of power corrections.

\subsection{Tests of Factorization in $B \rightarrow \pi K, \pi \pi$}

The factorization formula for $B$ decays into two light mesons is more complicated because of the presence of the two types of contributions shown in the lower graph in Figure 5 . The finding that these two topologies contribute at the same power in $\Lambda_{\mathrm{QCD}} / m_{b}$ is nontrivial 45 and relies on the heavy-quark scaling law $F^{B \rightarrow L}(0) \sim m_{b}^{-3 / 2}$ for heavy-tolight form factors, 5960.61 which is established less rigorously than the corresponding scaling law for heavy-to-heavy form factors. In the QCD factorization approach the kernels $T_{i j}^{I}(\mu)$ are of order unity, whereas the kernels $T_{i j}^{I I}(\mu)$ contribute first at order $\alpha_{s}$. Numerically, the latter ones give corrections of about 10-20\% with respect to the leading terms. Therefore, the scaling laws that form the basis of the QCD factorization formula appear to work well empirically.

The factorization formula for $B$ decays into two light mesons can be tested best by using decays that have negligible amplitude interference. In that way any sensitivity to the value of the weak phase $\gamma$ is avoided. For a complete theoretical control over charmless hadronic decays one must control the magnitude of the tree topologies, the magnitude of the penguin topologies, and the relative strong-interaction phases between trees and penguins. It is important that these three key features can be tested separately. Once these tests are conclusive (and assuming they are successful), factorization can be used to constrain the parameters of the unitarity triangle.

\section{Magnitude of the Tree Amplitude}

The magnitude of the leading $B \rightarrow \pi \pi$ tree amplitude can be probed in the decays $B^{ \pm} \rightarrow \pi^{ \pm} \pi^{0}$, which to an excellent approximation do not receive any penguin contributions. The QCD factorization approach makes an absolute prediction for the corresponding branching ratio, 15

$$
\begin{aligned}
& \operatorname{Br}\left(B^{ \pm} \rightarrow \pi^{ \pm} \pi^{0}\right)=\left[\frac{\left|V_{u b}\right|}{0.0035} \frac{F_{0}^{B \rightarrow \pi}(0)}{0.28}\right]^{2} \\
& \left.\times\left[5.3_{-0.4}^{+0.8} \text { (pars. }\right) \pm 0.3 \text { (power) }\right] \cdot 10^{-6},
\end{aligned}
$$

which compares well with the experimental result $(5.7 \pm 1.5) \times 10^{-6}$ (see the table in Figure 8 for a compilation of the experimental data on charmless hadronic decays). The theoretical uncertainties quoted are due to input parameter variations and the modeling of power corrections. An additional large uncertainty comes from the present error on $\left|V_{u b}\right|$ and the semileptonic $B \rightarrow \pi$ form factor. The sensitivity to these quantities can be eliminated by taking the ratio

$$
\begin{gathered}
\frac{\Gamma\left(B^{ \pm} \rightarrow \pi^{ \pm} \pi^{0}\right)}{d \Gamma\left(\bar{B}^{0} \rightarrow \pi^{+} l^{-} \bar{\nu}\right) /\left.d q^{2}\right|_{q^{2}=0}} \\
=3 \pi^{2} f_{\pi}^{2} \underbrace{\left|a_{1}^{(\pi \pi)}+a_{2}^{(\pi \pi)}\right|^{2}}_{1.33_{-0.11}^{+0.20} \text { (pars.) } \pm 0.07 \text { (power) }}=\left(0.68_{-0.06}^{+0.11}\right) \mathrm{GeV}^{2} .
\end{gathered}
$$

This prediction includes a sizeable $(\sim 25 \%)$ contribution of the hard-scattering term in the factorization formula (the last graph in Figure 5). Unfortunately, this ratio has not yet been measured experimentally.

\section{Magnitude of the T/P Ratio}

The magnitude of the leading $B \rightarrow \pi K$ penguin amplitude can be probed in the decays 
$B^{ \pm} \rightarrow \pi^{ \pm} K^{0}$, which to an excellent approximation do not receive any tree contributions. Combining it with the measurement of the tree amplitude just described, a treeto-penguin ratio can be determined via the relation

$\varepsilon_{\exp }=\left|\frac{T}{P}\right|=\tan \theta_{C} \frac{f_{K}}{f_{\pi}}\left[\frac{2 \operatorname{Br}\left(B^{ \pm} \rightarrow \pi^{ \pm} \pi^{0}\right)}{\operatorname{Br}\left(B^{ \pm} \rightarrow \pi^{ \pm} K^{0}\right)}\right]^{\frac{1}{2}}$.

The present experimental value $\varepsilon_{\exp }=$ $0.223 \pm 0.034$ is in good agreement with the theoretical prediction $\varepsilon_{\mathrm{th}}=0.24 \pm$ 0.04 (pars.) \pm 0.04 (power) $\pm 0.05\left(V_{u b}\right), 45$ which is independent of form factors but proportional to $\left|V_{u b} / V_{c b}\right|$. This is a highly nontrivial test of the QCD factorization approach. Recall that, when the first measurements of charmless hadronic decays appeared, several authors remarked that the penguin amplitudes were much larger than expected based on naive factorization models. We now see that QCD factorization reproduces naturally (i.e., for central values of all input parameters) the correct magnitude of the tree-to-penguin ratio. This observation also shows that there is no need to supplement the QCD factorization predictions in an ad hoc way by adding enhanced phenomenological penguin amplitudes, such as the "nonperturbative charming penguins" introduced some time ago.62 (The effects of charming penguins can be parameterized in terms of a "bag parameter" $\hat{B}_{1}=$ $(0.13 \pm 0.02) e^{i(188 \pm 82)^{\circ}}$ fitted to the data on charmless decays 63 By definition, this parameter contains the contribution from the perturbative charm loop, which is calculable in QCD factorization. Using the factorization approach one finds that $\hat{B}_{1}^{\text {fact }}=$ $\left(0.09_{-0.02-0.02}^{+0.03+0.04}\right) e^{i(185 \pm 3 \pm 21)^{\circ}}$, where the errors are due to input parameter variations and the estimate of power corrections. The perturbative contribution to the central value is 0.08 ; the remaining 0.01 is mainly due to weak annihilation. Hence, within errors QCD factorization can account for the "charming penguin bag parameter", which is in fact dominated by short-distance physics.)

\section{Strong Phase of the T/P Ratio}

The QCD factorization approach predicts that strong-interaction phases in most charmless hadronic $B$ decays are parametrically suppressed in the heavy-quark limit, i.e., $\phi_{\mathrm{st}}=O\left[\alpha_{s}\left(m_{b}\right), \Lambda_{\mathrm{QCD}} / m_{b}\right]$. This implies small direct CP asymmetries since, e.g., $A_{\mathrm{CP}}\left(\pi^{+} K^{-}\right) \simeq-2\left|\frac{T}{P}\right| \sin \gamma \sin \phi_{\mathrm{st}}$. The suppression results as a consequence of systematic cancellations of soft contributions, which are missed in phenomenological models of final-state interactions. In many other schemes the strong-interaction phases are predicted to be much larger, and therefore larger CP asymmetries are expected. Table 1 shows that first experimental data provide no evidence for large direct $\mathrm{CP}$ asymmetries in $B \rightarrow \pi K$ decays. However, the errors are still too large to draw a definitive conclusion that would allow us to distinguish between different theoretical predictions.

\subsection{Remarks on Sudakov Logarithms}

In recent years, $\mathrm{Li}$ and collaborators have proposed an alternative scheme for calculating nonleptonic $B$ decay amplitudes based a perturbative hard-scattering approach. 35.36 From a conceptual point of view, the main difference between QCD factorization and this so-called pQCD approach lies in the latter's assumption that Sudakov form factors effectively suppress soft-gluon exchange in diagrams such as those shown in Figure 5. As a result, the $B \rightarrow \pi$ and $B \rightarrow K$ form factors are assumed to be perturbatively calculable. This changes the counting of powers of $\alpha_{s}$. In particular, the nonfactorizable gluon-exchange diagrams included in the QCD factorization approach, which are crucial in order to cancel the scale and schemedependence in the predictions for the decay amplitudes, are formally of order $\alpha_{s}^{2}$ in the 
Table 1. Direct $\mathrm{CP}$ asymmetries in $B \rightarrow \pi K$ decays

\begin{tabular}{l|c|ccc}
\hline & Experiment & \multicolumn{3}{|c}{ Theory } \\
& 64.65.66.67 & Beneke et al. 45 & Keum et al.36 & Ciuchini et al.62 \\
\hline$A_{\mathrm{CP}}\left(\pi^{+} K^{-}\right)(\%)$ & $-4.8 \pm 6.8$ & $5 \pm 9$ & -18 & $\pm(17 \pm 6)$ \\
$A_{\mathrm{CP}}\left(\pi^{0} K^{-}\right)(\%)$ & $-9.6 \pm 11.9$ & $7 \pm 9$ & -15 & $\pm(18 \pm 6)$ \\
$A_{\mathrm{CP}}\left(\pi^{-} \bar{K}^{0}\right)(\%)$ & $-4.7 \pm 13.9$ & $1 \pm 1$ & -2 & $\pm(3 \pm 3)$ \\
\hline
\end{tabular}

pQCD scheme and consequently are left out. Thus, to the considered order there are no loop graphs that could give rise to stronginteraction phases in that scheme. (However, large phases are claimed to arise from on-shell poles of massless propagators in tree diagrams 36 Because these phases are dominated by soft physics, the prediction of large direct $\mathrm{CP}$ asymmetries in the pQCD approach rests on assumptions that are strongly model dependent.)

The assumption of Sudakov suppression in hadronic $B$ decays is questionable, because the relevant scale $Q^{2} \sim m_{b} \Lambda_{\mathrm{QCD}} \sim 1 \mathrm{GeV}^{2}$ is not that large for realistic $b$-quark masses. Indeed, one finds that the pQCD calculations are very sensitive to details of the $p_{\perp}$ dependence of the wave functions. 68 This sensitivity to hadronic physics invalidates the original assumption of an effective suppression of soft contributions. (The argument just presented leaves open the conceptual question whether Sudakov logarithms are relevant in the asymptotic limit $m_{b} \rightarrow \infty$. This question has not yet been answered in a satisfactory way.)

\section{Constraints in the $(\bar{\rho}, \bar{\eta})$ Plane}

The QCD factorization approach, combined with a conservative estimate of power corrections, offers several new strategies to derive constraints on CKM parameters 45 Some of these strategies will be illustrated below. Note that the applications of QCD factorization are not limited to computing branching ratios. The approach is also useful in combination with other ideas based on flavor symmetries and amplitude relations. In this way, strategies can be found for which the residual hadronic uncertainties are simultaneously suppressed by three small parameters, since they vanish in the heavy-quark limit $\left(\sim \Lambda_{\mathrm{QCD}} / m_{b}\right)$, the limit of $\mathrm{SU}(3)$ flavor symmetry $\left(\sim\left(m_{s}-m_{q}\right) / \Lambda_{\mathrm{QCD}}\right)$, and the large- $N_{c}$ limit $\left(\sim 1 / N_{c}\right)$.

\subsection{Extraction of $\gamma$ with Minimal Theory Input}

Some years ago, Rosner and the present author have derived a bound on $\gamma$ by combining measurements of the ratios $\varepsilon_{\exp }=|T / P|$ and $R_{*}=\frac{1}{2} \Gamma\left(B^{ \pm} \rightarrow \pi^{ \pm} K^{0}\right) / \Gamma\left(B^{ \pm} \rightarrow\right.$ $\left.\pi^{0} K^{ \pm}\right)$with the fact that for an arbitrary strong-interaction phase $-1 \leq \cos \phi_{\text {st }} \leq$ 1.38 The model-independent observation that $\cos \phi_{\text {st }}=1$ up to second-order corrections to the heavy-quark limit can be used to turn this bound into a determination of $\gamma$ (once $\left|V_{u b}\right|$ is known). The resulting constraints in the $(\bar{\rho}, \bar{\eta})$ plane, obtained under the conservative assumption that $\cos \phi_{\text {st }}>0.8$ (corresponding to $\left|\phi_{\mathrm{st}}\right|<37^{\circ}$ ), are shown in Figure 6 for several illustrative values of the ratio $R_{*}$. Note that for $0.8<R_{*}<1.1$ (the range preferred by the SM) the theoretical uncertainty reflected by the widths of the bands is smaller than for any other constraint on $(\bar{\rho}, \bar{\eta})$ except for the one derived from the $\sin 2 \beta_{\psi K}$ measurement. With present data the SM is still in good shape, but it will be interesting to see what happens when the experimental errors are reduced. 


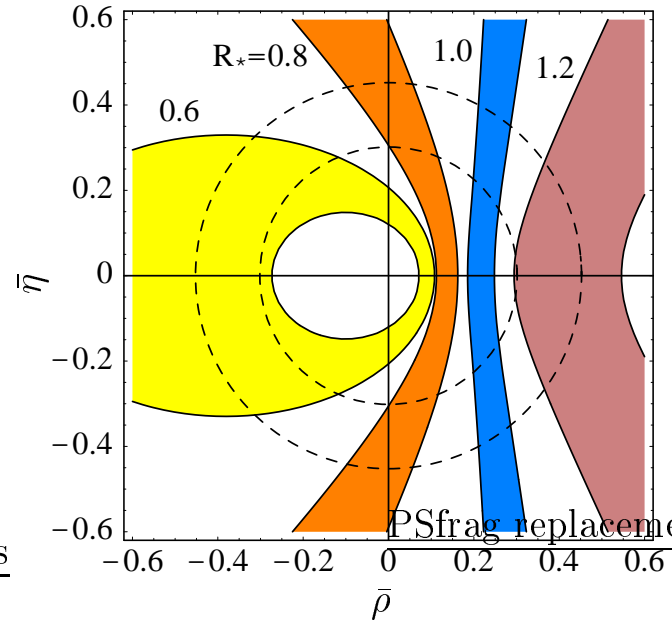

Figure 6. Allowed regions in the $(\bar{\rho}, \bar{\eta})$ plane corresponding to $\varepsilon_{\exp }=0.22$ and different values of the ratio $R_{*}$. The widths of the bands reflect the theoretical uncertainty. The current experimental values are $\varepsilon_{\exp }=0.22 \pm 0.03$ and $R_{*}=0.71 \pm 0.14$.

\subsection{Determination of $\sin 2 \alpha$}

With the help of QCD factorization it is possible to control the "penguin pollution" in the time-dependent $\mathrm{CP}$ asymmetry in $B \rightarrow$ $\pi^{+} \pi^{-}$decays, defined such that $S_{\pi \pi}=\sin 2 \alpha$. $[1+O(P / T)]$. This is illustrated in Figure 7, which shows the constraints imposed by a measurement of $S_{\pi \pi}$ in the $(\bar{\rho}, \bar{\eta})$ plane. Even a result for $S_{\pi \pi}$ with large experimental errors would imply a useful constraint on the unitarity triangle. A first, preliminary measurement of the asymmetry has been presented by the BaBar Collaboration at this conference.67 Their result is $S_{\pi \pi}=$ $0.03_{-0.56}^{+0.53} \pm 0.11$.

\subsection{Global Fit to the $B \rightarrow \pi K, \pi \pi$ Branching Ratios}

Various ratios of $\mathrm{CP}$-averaged $B \rightarrow \pi K, \pi \pi$ branching fractions exhibit a strong dependence on $\gamma$ and $\left|V_{u b}\right|$, or equivalently, on the parameters $\bar{\rho}$ and $\bar{\eta}$ of the unitarity triangle. From a global analysis of the experimental data it is possible to derive constraints in the

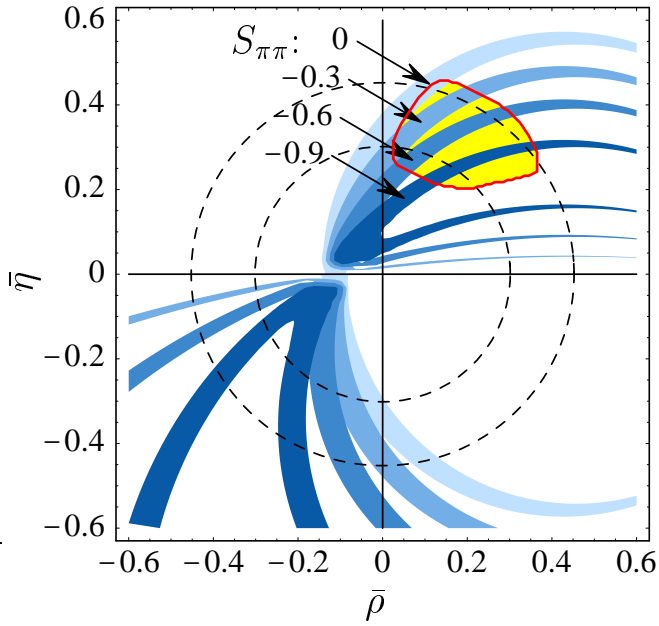

Figure 7. Allowed regions in the $(\bar{\rho}, \bar{\eta})$ plane corresponding to different values of $S_{\pi \pi}$. The widths of the bands reflect the theoretical uncertainty. The corresponding bands for positive $S_{\pi \pi}$ are obtained by a reflection about the $\bar{\rho}$ axis. The bounded light area shows the allowed region obtainef from the standard analysis of the unitarity triangle.t

$(\bar{\rho}, \bar{\eta})$ plane in the form of regions allowed at various confidence levels. The results of such an analysis are shown in Figure 8. The best fit of the QCD factorization theory to the data yields an excellent $\chi^{2} / n_{\text {dof }}$ of about 0.5 . (We disagree with the implementation of our approach presented in recent work by Ciuchini et al.63 and, in particular, with the numerical results labeled "BBNS" in Table II of that paper, which led the authors to the conclusion that the "theory of QCD factorization $\ldots$ is insufficient to fit the data". Even restricting $(\bar{\rho}, \bar{\eta})$ to lie within the narrow ranges adopted by these authors, one finds parameter sets for which QCD factorization fits the data with a $\chi^{2} / n_{\text {dof }}$ of less than 1.5 .)

The results of this global fit are compatible with the standard CKM fit using semileptonic decays, $K-\bar{K}$ mixing, and $B-\bar{B}$ mixing $\left(\left|V_{u b}\right|,\left|V_{c b}\right|, \epsilon_{K}, \Delta m_{d}, \Delta m_{s}, \sin 2 \beta_{\psi K}\right)$, although the fit prefers a slightly larger value of $\gamma$ and a smaller value of $\left|V_{u b}\right|$. The combination of the results from rare hadronic $B$ decays with $\left|V_{u b}\right|$ from semileptonic decays 


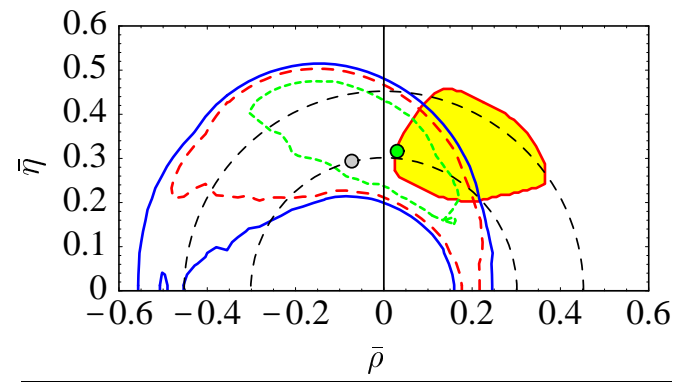

\begin{tabular}{l|c|c}
\hline Decay Mode & Best Fit & Exp. Average \\
\hline$B^{0} \rightarrow \pi^{+} \pi^{-}$ & 4.6 & $4.1 \pm 0.9$ \\
$B^{ \pm} \rightarrow \pi^{ \pm} \pi^{0}$ & 5.3 & $5.7 \pm 1.5$ \\
\hline$B^{0} \rightarrow \pi^{\mp} K^{ \pm}$ & 17.9 & $17.3 \pm 1.5$ \\
$B^{ \pm} \rightarrow \pi^{0} K^{ \pm}$ & 11.3 & $12.0 \pm 1.6$ \\
$B^{ \pm} \rightarrow \pi^{ \pm} K^{0}$ & 17.7 & $17.2 \pm 2.6$ \\
$B^{0} \rightarrow \pi^{0} K^{0}$ & 7.1 & $10.4 \pm 2.6$ \\
\hline
\end{tabular}

Figure 8. 95\% (solid), 90\% (dashed) and 68\% (shortdashed) confidence level contours in the $(\bar{\rho}, \bar{\eta})$ plane obtained from a global fit of QCD factorization results to the $\mathrm{CP}$-averaged $B \rightarrow \pi K, \pi \pi$ branching fractions. The dark dot shows the overall best fit; the light dot indicates the best fit for the default parameter set. The table compares the best fit values for the branching fraction (in units of $10^{-6}$ ) with the world average data.66.69. 70

excludes $\bar{\eta}=0$ at $95 \%$ confidence level, thus showing first evidence for the existence of a CP-violating phase in the bottom sector. Very soon, when the data become more precise, this will provide a powerful test of the CKM paradigm.

\section{Outlook}

The field of $B$ physics and $\mathrm{CP}$ violation is more lively and fascinating than ever. This year's discovery of $\mathrm{CP}$ violation in the $B$ system, combined with the recent discovery of direct $\mathrm{CP}$ violation in $K$ decays, are outstanding achievements and a triumph for the SM. They establish the CKM mechanism as the dominant source of $\mathrm{CP}$ violation in hadronic weak decays.

Yet, searches for deviations form the CKM paradigm are well motivated and must be continued with ever higher level of pre- cision. Some key measurements that can be performed in the near future include the observation of $B_{s}-\bar{B}_{s}$ mixing, the measurement of the CP-violating phase $\gamma$ in the bottom sector, and the discovery of the timedependent CP asymmetry in $B \rightarrow \pi^{+} \pi^{-}$decays. On the longer term, the main focus of $B$ physics should be on a systematic, detailed study of rare decay processes.

The QCD factorization approach provides the theoretical framework for a systematic analysis of hadronic and radiative exclusive $B$ decay amplitudes based on the heavyquark expansion. This theory has already passed successfully several nontrivial tests, and will be tested more thoroughly with more precise data. Ultimately, this may lead to theoretical control over a vast variety of exclusive $B$ decays, giving us new constraints on the unitarity triangle.

If the CKM mechanism remains to stand ever more precise experimental test we will be facing a new decoupling problem, whose resolution may be linked to whatever New Physics there is to discover at the $\mathrm{TeV}$ scale.

\section{Acknowledgments}

I wish to thank the organizers of LP01 for the invitation to deliver this talk and for their support. I am grateful to T. Becher, M. Beneke, G. Buchalla, Y. Grossman, A. Kagan, B. Pecjak, A. Petrov, J. Rosner, and C. Sachrajda for many enjoyable collaborations on work relevant to this talk. This work was supported in part by the National Science Foundation.

\section{References}

1. For a recent appraisal, see: H. R. Quinn, hep-ph/0110050.

2. B. Aubert et al. [BaBar Collaboration], Phys. Rev. Lett. 87, 091801 (2001).

3. K. Abe et al. [Belle Collaboration], Phys. Rev. Lett. 87, 091802 (2001). 
4. A. Höcker, H. Lacker, S. Laplace and F. Le Diberder, Eur. Phys. J. C 21, 225 (2001); for an update, see: http://www.slac.stanford.edu/ raplace/ckmfitter.html.

5. See, e.g.: R. Fleischer and T. Mannel, Phys. Lett. B 506, 311 (2001).

6. S. Bergmann and G. Perez, JHEP 0008, 034 (2000).

7. A. L. Kagan and M. Neubert, Phys. Lett. B 492, 115 (2000).

8. J. P. Silva and L. Wolfenstein, Phys. Rev. D 63, 056001 (2001).

9. G. Eyal, Y. Nir and G. Perez, JHEP 0008, 028 (2000).

10. Z. z. Xing, hep-ph/0008018.

11. G. Barenboim, F. J. Botella and O. Vives, Phys. Rev. D 64, 015007 (2001); Nucl. Phys. B 613, 285 (2001).

12. P. Ball, J. M. Frère and J. Matias, Nucl. Phys. B 572, 3 (2000).

13. P. Ball and R. Fleischer, Phys. Lett. B 475, 111 (2000).

14. S. Bergmann and G. Perez, hepph/0103299; JHEP 0008, 034 (2000).

15. A. Ali and E. Lunghi, hep-ph/0105200.

16. A. J. Buras, P. Gambino, M. Gorbahn, S. Jager and L. Silvestrini, Phys. Lett. B 500, 161 (2001).

17. A. J. Buras and R. Buras, Phys. Lett. B 501, 223 (2001); A. J. Buras and R. Fleischer, hep-ph/0104238.

18. Y. Grossman and M. P. Worah, Phys. Lett. B 395, 241 (1997); Y. Grossman, G. Isidori and M. P. Worah, Phys. Rev. D 58, 057504 (1998).

19. A. L. Kagan and M. Neubert, Phys. Rev. D 58, 094012 (1998).

20. Y. Grossman, M. Neubert and A. L. Kagan, JHEP 9910, 029 (1999).

21. R. Fleischer and J. Matias, Phys. Rev. D 61, 074004 (2000).

22. G. Burdman, Phys. Rev. D 57, 4254 (1998); G. Burdman and G. Hiller, Phys. Rev. D 63, 113008 (2001).

23. A. Ali, P. Ball, L. T. Handoko and
G. Hiller, Phys. Rev. D 61, 074024 (2000).

24. M. Beneke, T. Feldmann and D. Seidel, Nucl. Phys. B 612, 25 (2001).

25. G. Buchalla and A. J. Buras, Phys. Rev. D 54, 6782 (1996).

26. D. Atwood, I. Dunietz and A. Soni, Phys. Rev. Lett. 78, 3257 (1997).

27. I. Dunietz and R. G. Sachs, Phys. Rev. D 37, 3186 (1988) [Erratum-ibid. D 39, 3515 (1988)]; I. Dunietz, Phys. Lett. B 427, 179 (1998).

28. M. Gronau and D. London, Phys. Rev. Lett. 65, 3381 (1990).

29. A. E. Snyder and H. R. Quinn, Phys. Rev. D 48, 2139 (1993).

30. M. Gronau, J. L. Rosner and D. London, Phys. Rev. Lett. 73, 21 (1994); O. F. Hernandez, D. London, M. Gronau and J. L. Rosner, Phys. Lett. B 333, 500 (1994); M. Gronau, O. F. Hernandez, D. London and J. L. Rosner, Phys. Rev. D 50, 4529 (1994).

31. A. J. Buras and L. Silvestrini, Nucl. Phys. B 569, 3 (2000).

32. For a review, see: R. Fleischer, Int. J. Mod. Phys. A 12, 2459 (1997).

33. M. Beneke, G. Buchalla, M. Neubert and C. T. Sachrajda, Phys. Rev. Lett. 83, 1914 (1999).

34. M. Beneke, G. Buchalla, M. Neubert and C. T. Sachrajda, Nucl. Phys. B 591, 313 (2000).

35. C. H. Chang and H. n. Li, Phys. Rev. D 55, 5577 (1997); T. W. Yeh and H. n. Li, Phys. Rev. D 56, 1615 (1997).

36. Y. Keum, H. Li and A. I. Sanda, Phys. Lett. B 504, 6 (2001); Phys. Rev. D 63, 054008 (2001); Y. Keum and H. Li, Phys. Rev. D 63, 074006 (2001).

37. R. Fleischer and T. Mannel, Phys. Rev. D 57, 2752 (1998).

38. M. Neubert and J. L. Rosner, Phys. Lett. B 441, 403 (1998); M. Neubert, JHEP 9902, 014 (1999).

39. C. W. Bauer, S. Fleming, D. Pirjol and 
I. W. Stewart, Phys. Rev. D 63, 114020 (2001); C. W. Bauer and I. W. Stewart, Phys. Lett. B 516, 134 (2001); C. W. Bauer, D. Pirjol and I. W. Stewart, hep-ph/0109045.

40. J. D. Bjorken, Nucl. Phys. Proc. Suppl. 11, 325 (1989).

41. M. J. Dugan and B. Grinstein, Phys. Lett. B 255, 583 (1991).

42. H. D. Politzer and M. B. Wise, Phys. Lett. B 257, 399 (1991).

43. M. Neubert and A. A. Petrov, Phys. Lett. B 519, 50 (2001).

44. H. Y. Cheng and K. C. Yang, Phys. Rev. D 64, 074004 (2001).

45. M. Beneke, G. Buchalla, M. Neubert and C. T. Sachrajda, Nucl. Phys. B 606, 245 (2001).

46. A. Khodjamirian, Nucl. Phys. B 605, 558 (2001).

47. C. N. Burrell and A. R. Williamson, Phys. Rev. D 64, 034009 (2001).

48. T. Becher, M. Neubert and B. D. Pecjak, hep-ph/0102219.

49. C. W. Bauer, D. Pirjol and I. W. Stewart, hep-ph/0107002.

50. Z. Ligeti, M. Luke and M. B. Wise, Phys. Lett. B 507, 142 (2001).

51. M. Diehl and G. Hiller, JHEP 0106, 067 (2001).

52. E. von Toerne (CLEO Collaboration), talk at the International Europhysics Conference on High-Energy Physics, Budapest, Hungary, July 2001.

53. K. Abe et al. [Belle Collaboration], hep$\mathrm{ex} / 0107048$.

54. S. W. Bosch and G. Buchalla, hep$\mathrm{ph} / 0106081$.

55. T. E. Coan et al. [CLEO Collaboration], Phys. Rev. Lett. 84, 5283 (2000).

56. Y. Ushiroda [Belle Collaboration], hep$\mathrm{ex} / 0104045$.

57. J. Nash [BaBar Collaboration], talk at this conference.

58. A. L. Kagan and M. Neubert, hep$\mathrm{ph} / 0110078$.
59. V. L. Chernyak and I. R. Zhitnitsky, Nucl. Phys. B 345, 137 (1990).

60. A. Ali, V. M. Braun and H. Simma, Z. Phys. C 63, 437 (1994).

61. E. Bagan, P. Ball and V. M. Braun, Phys. Lett. B 417, 154 (1998); P. Ball and V. M. Braun, Phys. Rev. D 58, 094016 (1998).

62. M. Ciuchini, E. Franco, G. Martinelli and L. Silvestrini, Nucl. Phys. B 501, 271 (1997); M. Ciuchini, E. Franco, G. Martinelli, M. Pierini and L. Silvestrini, Phys. Lett. B 515, 33 (2001).

63. M. Ciuchini, E. Franco, G. Martinelli, M. Pierini and L. Silvestrini, hep$\mathrm{ph} / 0110022$.

64. S. Chen et al. [CLEO Collaboration], Phys. Rev. Lett. 85, 525 (2000).

65. K. Abe et al. [Belle Collaboration], Phys. Rev. D 64, 071101 (2001).

66. B. Aubert et al. [BaBar Collaboration], hep-ex/0105061.

67. B. Aubert et al. [BaBar Collaboration], hep-ex/0107074.

68. S. Descotes-Genon and C. T. Sachrajda, hep-ph/0109260.

69. D. Cronin-Hennessy et al. [CLEO Collaboration], Phys. Rev. Lett. 85, 515 (2000).

70. K. Abe et al. [Belle Collaboration], Phys. Rev. Lett. 87, 101801 (2001). 